\title{
Rapid tranquillisation: a questionnaire survey of practice
}

\author{
Deborah Simpson and lan Anderson
}

\begin{abstract}
A poetal survey of the drug treatment of behovioural emergencies by senior registrars and consultants in peychiatry revealed that $90 \%$ would initially use a nondepot antipoychotic (including $24 \%$ who would combine It with a benzodiczepine if the sthuation warranted), and $10 \%$ the short-acting depot, zuclopenthixol acetate, with onother antipoychotic or a benzodiczepine, or both. The choice was made from a restricted range of drugs, with haloperidel being the moet popular antipsychotic and cliczepan the most popular benzodiazepine. Hatf of respondents ald not consider Brtitsh National Formulary maximum dose recommendations to be usetul for rapid tranquilitsation and many would exceed them. A substantilat minortly did not consider that adequate resuscliation was avallable at their unit and deficiencies were revealed in the training of junior doctors and in the ouclit of rapid tranquillisation. Very fow poychiatrists reported that their units had written guidelines.
\end{abstract}

The drug management of acutely disturbed psychotic patients (rapid tranquillisation) is an important aspect of psychiatric treatment but the guidance available from standard texts is limited and provides little practical help. Most psychiatrists learn from experience and advice from more senior doctors (i.e. senior registrars and consultants). Recent surveys of junior doctors' practice (Pllowsky et al, 1992) or consultant opinion (Cunnane, 1994) suggest a wide variation in regimes used, some potentially dangerous because of the use of high doses of drugs. There has been recent concern about the use of high dose antipsychotic medication, including in emergency situations (Thompson, 1994).

\section{The study}

Questionnaires were sent to 100 consultants and senior registrars in psychiatry in 12 psychiatric hospitals in the Greater Manchester area including mental handicap and forensic units. The questionnaire presented a brief vignette of an antipsychotic-naive man with disturbed, violent behaviour for whom emergency drug treatment for tranquillisation was necessary. Information was requested about the psychiatrist's choice of drugs, dosage and views on related issues including resuscitation and training. If the questionnaire was not returned within four weeks a second one was sent.

Differences between groups of psychiatrists were compared using $\chi^{2}$ and $t$-tests.

\section{Findings}

Sixty-nine questionnaires were returned. Two were not completed (one psychiatrist refused and one had retired) leaving 67 completed forms $(67 \%)$. The results are presented as percentages of the completed questionnaires.

\section{The sample}

Sixty per cent were consultants and $40 \%$ were senior registrars. Current posts were $57 \%$ in general adult psychiatry (7\% with a special interest), $19 \%$ in forensic psychiatry, $12 \%$ in old age psychiatry and $12 \%$ full-time in other specialities (addictions, mental handicap and rehabilitation). Ninety-four per cent treated inpatients and $76 \%$ had given or advised on rapid tranquillisation in the last six months. Consultants had practised psychiatry for a mean of 16 years (range 7-30 years) and senior registrars for seven years (range 4-13 years).

\section{Drug use}

For initial management the majority (90\%) of psychiatrists would use a single, non-depot, antipsychotic. Twenty-four per cent would combine this with a benzodiazepine $13 \%$ from the first dose and another $10 \%$ if the situation was extreme or not improving after an antipsychotic alone). Ten per cent would use the short-acting depot, zuclopenthixol acetate (Clopixol Acuphase), always in combination with another antipsychotic or a benzodiazepine, or frequently both. Only three non-depot antipsychotics were chosen, haloperidol (49\%), chlorpromazine (34\%) and droperidol (15\%), and two benzodiazepines, diazepam (19\%) and lorazepam (13\%). If the firstline antipsychotic drugs had to be given parenterally, $70 \%$ would give it intramuscularly, $9 \%$, intravenously, $10 \%$ by either parenteral route (10\% did not specify). Diazepam was nearly 
Table 1. Use of specific treatments for rapid tranquillisation

\begin{tabular}{llll}
\hline & \multicolumn{3}{l}{ Would usefor rapld tranquillisallion? } \\
\cline { 2 - 4 } Drug & Yes (\%) & No (\%) & Not stated (\%) \\
\hline Benzodiczepines & 91 & 6 & 3 \\
Methotimeprazine & 51 & 49 & - \\
Zuclopenthixol & 82 & 18 & - \\
$\quad$ acetate & & & \\
Depot antipsychotics & 54 & 45 & 1 \\
Paraldehyde & 28 & 69 & 3 \\
Barblturates & 21 & 78 & 1 \\
ECT & 66 & 34 & - \\
\hline
\end{tabular}

always given intravenously and lorazepam intramuscularly. Drug administration regimes varied widely, from every half hour to eight hourly.

Most (84\%) gave drug properties (sedative qualities, potency, rapidity of action and relative safety) as the reason for the initial choice of medication, with $19 \%$ citing clinical experience instead or in addition. Seven per cent commented that use of zuclopenthixol acetate avoided repeated injections.

If the initial regime was unsuccessful a variety of options were proposed (the total exceeds $100 \%$ as many respondents gave more than one drug or alternative). The most popular response was to change antipsychotic (55\%). This nearly always included one of the first-line drugs (haloperidol, droperidol or chlorpromazine); $34 \%$ chose zuclopenthixol acetate and $3 \%$ chose methotrimeprazine. Changing the route of administration, dose or frequency of the first-line antipsychotic was advocated by $33 \%$. Adding in a benzodiazepine was chosen by $39 \%$ of respondents, combining different antipsychotics (not including zuclopenthixol acetate) by $4 \%$ and adding a long-acting depot by $6 \%$. Other infrequent suggestions included giving electroconvulsive therapy (ECT), paraldehyde or a barbiturate, reviewing mental state, a physical examination, the treatment of any akathisia and seeking a second opinion. One respondent could not concelve that the first-line treatment would not work.

Respondents were asked if they used specific antipsychotic preparations, routes of administration and less common or controversial agents. Selected results are shown in Table 1 . Twenty-two per cent of respondents stated that they would use intramuscular diazepam.

\section{Maximum doses}

Forty-five per cent of respondents believed that British National Formulary (1995) maximum doses are at least usually relevant for rapid tranquillisation, if only as a guide, but $48 \%$ believed that they are usually not relevant. Three per cent thought that they were only relevant for drug-naive patients and $4 \%$ gave no opinion. The median maximum doses of selected drugs that respondents would use are given in Table 2. Modal doses were the same as the medians in nearly all cases.

\section{Issues related to practice and training}

Respondents were asked to rate their satisfaction with drug and non-drug management of behavioural disturbance in their units on a scale of $0=$ not at all satisfied to $100=$ completely satisfied. Eighty-four per cent of psychiatrists answered this question (an additional 10\% stated it was not applicable to their situation). The mean satisfaction rating for drug treatment was 68.3 (s.d.=18.6. range 10-100) and for non-drug treatment was 61.3 (s.d.=24.4, range 0-100) indicating a wide range of views.

Forty-eight per cent reported that junior medical staff were trained in the use of drugs for rapid tranquillisation, $27 \%$ reported no training and $25 \%$ did not know/answer. Sixty-three per cent believed that staff were trained in the management of behavioural disturbance by non-drug measures (3\% commented that this applied only to some staff) while $27 \%$ reported no or little training and $10 \%$ did not know/answer.

Table 2. Maximum doses of selected drugs that respondents would give for rapid tranquillisation

\begin{tabular}{|c|c|c|c|c|}
\hline \multirow[b]{2}{*}{ Drug } & \multicolumn{2}{|c|}{ Oral administration } & \multicolumn{2}{|c|}{ Porenteral administration } \\
\hline & Single dose/mg" & Dose in 2Ahrs/mg' & Singlo doee/mg* & Dose in 2Ahrs/mg" \\
\hline $\begin{array}{l}\text { Haloperidol } \\
\text { Chlorpromazine } \\
\text { Droperidol } \\
\text { Dlazepam } \\
\text { Lorazepam } \\
\text { Paraldehyde }\end{array}$ & $\begin{array}{c}20 \text { (10-no limit) } \\
200(100-400) \\
20(10-40) \\
20(10-30) \\
4(2-10) \\
-\end{array}$ & $\begin{array}{c}120 \text { (40-no limit) } \\
1000(400-2000) \\
120(40-480) \\
60(10-120) \\
12(4-32) \\
-\end{array}$ & $\begin{array}{l}20 \text { (5-no limit) } \\
100(50-250) \\
20(5-30) \\
10(10-100) \\
4(1-5) \\
10(10-20)^{\dagger}\end{array}$ & $\begin{array}{c}80 \text { (30-no limit) } \\
500(200-1000) \\
80(5-140) \\
40(10-200) \\
8(2-24) \\
20(10-80)^{\dagger}\end{array}$ \\
\hline
\end{tabular}

-Doses are median (range)

'Doses are in $\mathrm{ml}$ 
Forty per cent of psychiatrists had access to an intensive care unit and the same percentage of psychiatrists reported the use of seclusion. When this was related to speciality it applied to $100 \%$ of forensic psychiatrists but only $25 \%$ of other psychiatrists $\left(\chi^{2}=26.219\right.$, d.f. $\left.=2, P<0.001\right)$.

Audit of the management of behavioural disturbance was reported by $37 \%$ of psychiatrists (79\% of forensic psychiatrists and $26 \%$ of others, $\chi^{2}=12.911$, d.f. $=2, P<0.01$ ). Only $15 \%$ of psychiatrists reported that their units had written guidelines for rapid tranquillisation $(18 \%$ did not know/answer). The percentage having written guidelines for the general management of behavioural disturbance was $30 \%$ with $22 \%$ not knowing/answering.

\section{Resuscitation}

Sixty per cent of psychiatrists believed that adequate resuscitation was available on their units, 19\% thought that it was inadequate and $21 \%$ did not know/answer or thought it not applicable. There was a trend for fewer senior registrars to be satisfied than consultants $(44 \% \mathrm{v}$. $70 \%, \chi^{2}=5.484$, d.f. $=2, P<0.06$ ).

\section{Comment}

On the whole psychiatrists chose their initial treatment from a limited range of drugs. As might be expected the majority started treatment with a single antipsychotic, although a quarter would combine this with a benzodiazepine if needed. Haloperidol was the most frequently chosen drug which contrasts with the preference for chlorpromazine in the survey by Cunnane (1994) and accords with the suggestion that high potency antipsychotics may be preferable in this situation to lessen the risk of hypotension (Anonymous. 1991). The combination with a benzodiazepine is consistent with current thinking that it is desirable to avoid very high antipsychotic drug doses when the immediate aim is sedation (Anonymous, 1991; Thompson, 1994). The choice, by a significant minority, of zuclopenthixol acetate as a first-line drug in an antipsychoticnaive patient, and usually in combination with another antipsychotic, is, however, worrying in view of the risk of administering a long acting drug in this situation. For this reason the Royal College of Psychiatrists' Consensus Statement (Thompson, 1994) recommends that it is only used in extreme circumstances when patients have not been previously exposed to antipsychotic drugs.

The changes in management adopted if the initial response was inadequate reveal no surprises although the high frequency of the use of zuclopenthixol acetate is notable and indicates its increasing acceptance for rapid tranquillisation by psychiatrists. It is also of interest that older treatments such as paraldehyde and barbiturates have fallen out of favour, probably as a reflection of the relatively high risk-benefit ratio of these drugs. A substantial percentage of psychiatrists would use diazepam intramuscularly in spite of poor absorption by this route (Greenblatt \& KochWeser, 1976).

About half of psychiatrists thought that maximum doses stated in the British National Formulary were not helpful for rapid tranquillisation. In practice the median and modal maximum daily antipsychotic doses psychiatrists would use were very close to the guidelines although they would use higher than recommended single doses of chlorpromazine (which are $75 \mathrm{mg}$ orally, at least initially, and $50 \mathrm{mg}$ parenterally). From this survey it appears that many psychiatrists believe that emergency situations may warrant higher doses of antipsychotics than recommended which is important in view of the increasing medicolegal implications of using doses in excess of British National Formulary guidelines. The British National Formulary does not give rapid tranquillisation as an indication for benzodiazepines and therefore provides no guidance on dosage in this situation. This is an omission which could usefully be rectified given the Royal College of Psychiatrists' endorsement of the use of benzodiazepines in emergency situations (Thompson. 1994).

Deficiencies are apparent when issues related to training and practice are considered. There is a lack of training of junior staff in rapid tranquillisation and audit is rarely carried out in specialities apart from forensic psychiatry, even though satisfaction with the management of behavioural disturbance is only moderately high. Written guidelines are rare and we would suggest that this is an area that deserves attention. As is well recognised, there is a deficiency in medium secure provision and only a minority of nonforensic psychiatrists had access to a psychiatric intensive care unit. Another area of concern is the quality of resuscitation available. Few psychiatric trainees have resuscitation training and when assessed their skills are found to be deficient (McNaughton et al, 1994). It is interesting that consultants and senior registrars tended to have different views on whether their units had adequate resuscitation. Senior registrars are usually more involved than consultants in emergency situations so arguably their view is more likely to be correct - it is therefore very worrying that only a minority of them were happy with resuscitation arrangements.

\section{Conclusion}

Most psychiatrists at senior registrar and consultant level use sensible drug regimes for rapid 
tranquillisation although the use of zuclopenthixol acetate for antipsychotic-naive patients is of concern. The British National Formulary could usefully include rapid tranquillisation as an indication for benzodiazepines. The development of rapid tranquillisation guidelines or protocols linked to audit may be one way of addressing the problems of insufficient training of junior doctors and the lack of audit in this area.

\section{Acknowledgements}

We would like to thank the psychiatrists who took the time and effort to complete a rather lengthy questionnaire.

\section{References}

ANONYMOUS (1991) Management of behavioural emergencles. Drugs and Therapeutics Bulletin, 29, 62-64.

BrTtSh MEdical association and the Pharmaceutical

SOCIETY (1995) Brttish National Formulary. No 29. London: BMA \& The Pharmaceutical Society.
CUNNANE, J. G. (1994) Drug management of disturbed behaviour by psychiatrists. Psychiatric Bulletin. 18. 138-139.

GREENBLATT, D. J. \& KoCH-WESER, J. (1976) Intramuscular injection of drugs. New England Journal of Medictne. 286, 542-546.

MCNAUGHTON, G., HALL, D. J. \& StaRK, C. (1994) Resuscitation skdils and doctors working in psychlatry. Psychiatric Bulletin, 18, 403-404.

PILOWSKY, L. S., RING, H., ShINE, P. J., et al (1992) Rapid tranquillisation: a survey of emergency prescribing in a general psychiatric hospital. British Joumal of Psychiatry. 160, 831-835.

THOMPSON, C. (1994) The use of high-dose antipsychotic medication. British Joumal of Psychiatry, 164, 448-458.

Deborah Simpson. Senior Registrar in Psychiatry, Rehabilitation Service, Harrop House, Prestwich Hospital, Bury New Road, Prestwich, Manchester M25 3BC; and "Ian Anderson, Senior Lecturer in Psychiatry. University of Manchester Department of Psychiatry, Rawnsley Building. Manchester Royal Infirmary, Oxford Road, Manchester M13 9WL

*Correspondence 